\title{
Medical Students' Knowledge, Attitudes, and Beliefs towards Firearm Ownership and Reporting
}

\author{
Aidan L Neustadtl MD \\ Cleveland Clinic Foundations \\ 9500 Euclid Ave, Cleveland \\ OH 44195 USA
}

\author{
Alicia Kaneb BS \\ Georgetown University School of Medicine \\ 3900 Reservoir Rd NW, District of Columbia \\ 20007 USA
}

Alan D Neustadtl PhD

University of Maryland, College Park

MD 20742 USA

David Milzman MD

University of Maryland School of Medicine

655 W Baltimore St S, Baltimore

MD 21201 USA

\begin{abstract}
"Red flag" firearm laws are a tool for physicians to report those considered at risk for violence to become temporarily or permanently ineligible to own firearms. To effectively identify those posing a risk to themselves or others, physicians must be well educated on firearm law and safety. In this study, medical students were queried on their knowledge, attitudes, and actions surrounding firearm ownership using a short survey. Results found that students overwhelmingly believe gun control is an important issue and the majority support gun control. However, less than $40 \%$ of students were able to pass a 10-question quiz on current gun laws. This survey found that medical students favor gun control but lack knowledge about gun safety. Despite medical student's passion about gun safety, there is a lack of knowledge about current gun laws. This discrepancy indicates a need to increase gun safety training and interventions during medical school.
\end{abstract}

Keywords: firearm, "red-flag", medical student, survey

\section{Introduction}

Mass shootings across the United States (US) have brought forth public outcry to reduce firearm access to persons at increased risk for violent behavior. Those flagged as at risk may be reported to third-parties such as psychiatrists and law enforcement to become temporarily or permanently ineligible to possess firearms. Groups that are likely to report red flag situations include teachers, therapists or counselors, and physicians. However, previous research estimated that almost a third of physicians were unsure about how to determine competence in patients applying for weapons permits and that physicians were not confident in their decisions regarding gun ownership. ${ }^{3}$

As debates over gun restrictions continue, the next generation of physicians may be tasked with combating gun violence and injury. For that reason, it is important that medical students are educated about firearm safety. Previous studies examining medical student training on firearm safety show that only $12 \%$ of students in their final year of medical school felt that they received extensive training, despite two-thirds reporting they had received some training. ${ }^{2}$ In the same study, $28 \%$ felt they were highly confident in talking to patients about firearm safety and $66 \%$ reported that they never or rarely counseled patients on firearm safety. ${ }^{2}$ A systematic review assessing education and curriculum of firearm safety in health care professionals (medical students, residents, and attendings) found only four papers 
describing outcomes from dedicated firearm safety training. ${ }^{8}$ This lack of training continues in residency training programs as well as in Continuing Medical Education for attending physicians. ${ }^{4}$

Understanding generational differences in beliefs and attitudes surrounding firearm ownership is important to understand how future physicians will look to address gun violence in their profession. A JAMA Pediatrics study found that youth beliefs on gun control were similar to their adult counterparts and tended to support legislation intended to increase public safety. ${ }^{10}$ They found that one-third were against guns in the home, while two-thirds believed that gun ownership is acceptable at least if certain safety conditions were met. ${ }^{10} 62 \%$ believed that mass shootings would decrease with enhanced gun control laws and respondents were most concerned about gun ownership for those with histories of violence, mental illness, and criminal records. ${ }^{10}$ A 2018 Marist poll found that 57\% of 18-29 year-olds believe stricter gun legislation should be an "immediate priority" of Congress. ${ }^{7} 48 \%$ of 18-29 year-olds also said a candidate's position on gun control would be a major factor in deciding to vote for them; however, respondents were split evenly among those who would vote for and against the sale of semi-automatic weapons. ${ }^{7}$

Little research exists on medical student's beliefs, attitudes, and actions surrounding firearm ownership and gun violence. Frank et al 2016, found that $14 \%$ of medical students in a national sample owned firearms, which is lower than the $30 \%$ estimate based on 2018 data from the General Social Survey for 18-34 year-olds who reported keeping a firearm in their home. ${ }^{2,9}$

Thecurrent study surveyed medical students primarily in the Washington, DC area concerning their knowledge, attitudes, and actions surrounding firearm ownership. Due to the apparent lack of gun violence education in medical school curricula, we hypothesized that medical students would not possess the comprehensive knowledge of current gun laws required to make informed decisions on gun ownership by their patients for increased public safety.

\section{Methods}

\subsection{Study Settings, Population, and Protocol}

The study population consisted of current, enrolled medical students in the United States and resulted in a sample of 102 respondents.

An online survey was distributed to medical students in the District of Columbia, Maryland, and Virginia region. This was done via emailing faculty at these schools as well as utilizing private Facebook groups consisting of current medical students.

\subsection{Survey Instrument}

A 37-question web-based survey was developed and revised by the principal investigators that took on average 10 to 15 minutes to complete. This survey was adapted from a previous study examining Emergency Department health care providers beliefs and knowledge on similar topics. ${ }^{6}$ The survey was intended to determine information about medical students: 1) knowledge about current firearm eligibility laws, 2) attitudes about reporting patients to third parties, 3) attitudes about firearm ownership/permits, and 4) demographic information. All responses were anonymous, and no incentives were offered for completion of the survey. To assess knowledge of current firearm eligibility laws, a 10question quiz about current restrictions and laws was included and a passing score was deemed 7/10 (70\%) correct answers. The questions were developed using information from the National Rifle Association-Institute for Legislative Action. ${ }^{1}$ To assess attitudes about reporting to third parties, medical students were presented nine clinical scenarios, and asked how likely they were to report the patient in the scenario to federal authorities. The responses were used to create an index that ranges from 1 to 57 with greater numbers representing a higher likelihood of reporting. To assess attitudes about firearm ownership/permits, the survey used questions included in the General Social Survey. ${ }^{9}$

\subsection{Data Analysis and Statistics}

Data for all cases were analyzed using Stata statistical software (V16.1). Categorical variables are reported as counts and percentages. Continuous variables are reported as means and standard deviations.

\section{Results}

A total of 102 medical students fully completed the survey. Response rate was not calculated as this was a public, webbased survey that was distributed broadly to medical faculty at medical schools to be shared with their students, thus making it difficult to assess the rate of response. The average age is 25 years old. 55\% were female and $65 \%$ were white $(n=102)$. Students at the Georgetown University School of Medicine (GUSOM) made up 70.6\% of participating respondents. $13.5 \%$ of students reported owning a gun. There was a bimodal distribution, with one-third of respondents 
being second-year students and one-third fourth-year students (Table 1). Over $60 \%$ of respondents consider themselves to be liberal and about 55\% identify as a Democrat, however, twenty-nine and thirty respondents did not respond to the political ideology and party questions, respectively (Figure 1).

\begin{tabular}{c|cc}
\multicolumn{1}{l}{ Table 1. } & \multicolumn{2}{c}{ Participant Demographics } \\
\hline \hline Age (mean +/- SD) & & $24.82+/-2.2$ \\
Gender (\%Male) & & $45.10 \%$ \\
Class (\%) & M1 & $19.60 \%$ \\
& M2 & $33.33 \%$ \\
& M3 & $13.73 \%$ \\
School (\%) & M4 & $33.33 \%$ \\
& GWU & $19.61 \%$ \\
& GUSOM & $70.59 \%$ \\
& Howard & $2.94 \%$ \\
Race (\%) & Other & $6.86 \%$ \\
& Asian & $20.00 \%$ \\
& Black or AA & $4 \%$ \\
& Hispanic & $2 \%$ \\
& Other & $9 \%$ \\
& White & $65 \%$ \\
Gun Owner (\%) & & $13.51 \%$
\end{tabular}

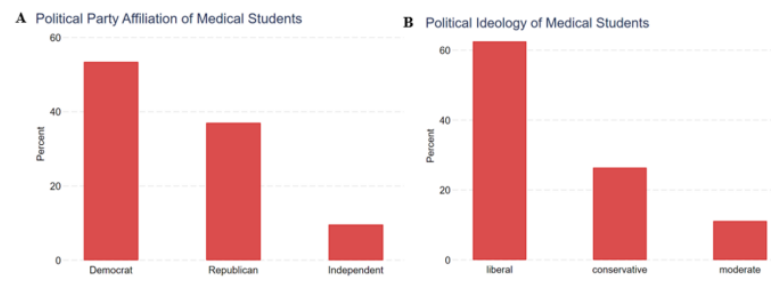

Fig. 1(A-B). Medical Students political party affiliation and political ideology. A) $53.4 \%$ of respondents reported as Democrats, while 37\% reported as Republicans, and 9.6\% reported as Independents. Twenty-nine people did not respond to this question. B) $62.5 \%$ of respondents considered they're ideology to be liberal, while $26.4 \%$ and $11.1 \%$ considered themselves to be conservative or moderate, respectively. Thirty people did not complete this question.

A 10-question quiz was used to assess knowledge on gun ownership eligibility and a "passing" score was defined as correctly answering 7 out of 10 questions. On average, medical students answered 5.9 questions correctly (mean 5.9, SD 1.7) and 36\% correctly answered 7 or more questions for a passing score (Figure 2A).
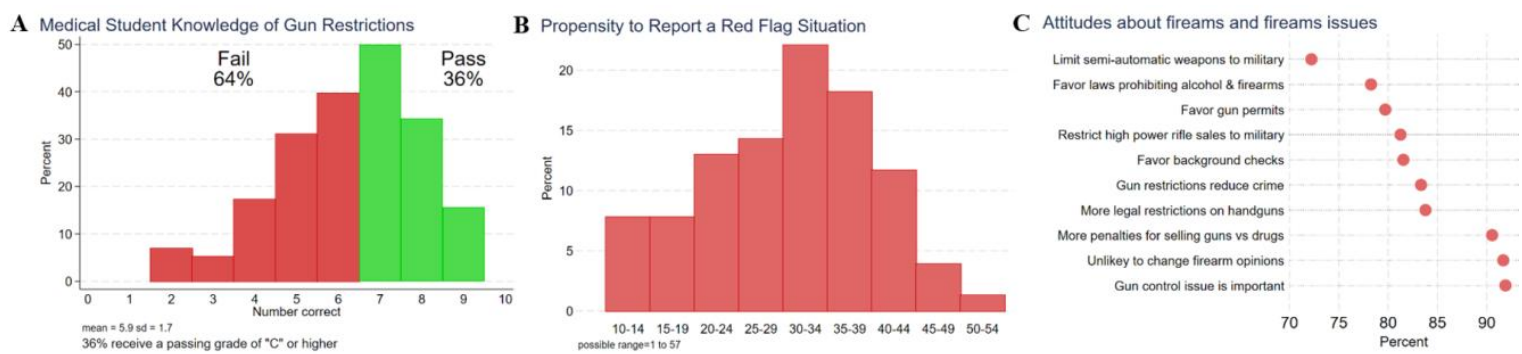

Fig. 2(A-C). Medical Student knowledge and attitude of firearm issues. A) On average, respondents answered 5.9 out of 10 questions on gun safety correctly and 36\% got at least $70 \%$ correct. B) Medical students were asked 9 different scenarios and whether they agree with reporting a patient to federal authorities on a scale from strongly agree to strongly disagree. Responses are weighted towards not reporting in most scenarios (mean 30.5, SD 9.6). C) Medical students' attitudes towards firearms and firearms issues are reported. 
Medical students were given 9 different clinical scenarios and asked how likely they were to report the patient in the scenario to federal authorities. Their responses were used to create an index that ranges between 1 and 57 with higher responses indicating more likely to report. Overall, their responses were weighted towards not reporting in most scenarios (mean 30.5, SD: 9.6; Figure 2B).

Greater than $90 \%$ reported that gun control is an important issue to them and that their attitudes towards firearms ownership are unlikely to change. Over $80 \%$ of respondents favor restricting high power rifle ownership to the military, requiring background checks for purchasing a firearm, and increasing legal restrictions on handguns. $84 \%$ believe that gun restrictions will reduce crime and $91 \%$ believe that penalties for violating gun sale laws should more severe than those for selling illicit drugs (Figure 2C).

\section{Discussion}

Slightly more than half of the medical students self-identified as Democrat (53.4\%), with $37 \%$ choosing Republican and $9 \%$ choosing independent. $62.5 \%$ of respondents also considered themselves to have a liberal ideology. This is opposed to previous surveys examining the political ideology of medical students that found $65 \%$ of medical students identified as conservative or very conservative and only $16 \%$ identified as liberal or very liberal. ${ }^{2}$ While the sample size in the current study is small and not nationally representative, it is possible given the predominantly urban location of the majority of respondents that this represents a more liberal-leaning group than would be expected across the entire country.

Gun ownership rates in our population were similar to previous samples of med students, despite differences in political ideology. Frank et al., 2006 found 14\% of nationally surveyed US medical students reported owning a gun. ${ }^{2}$ In comparison, $13.5 \%$ of students in our sample responded 'yes' to owning a gun, reflecting a nearly identical percentage. Frank et al., 2006 found that students located in the Mid-Atlantic had lower rates of gun ownership (5\%), but our sample of Mid-Atlantic students was over two times greater. ${ }^{2}$ This may be attributed to the small sample size in the current study or may represent a change in the broader geographic layout of gun ownership in the United States.

When looking at medical students' attitudes towards firearm issues, we found that students overwhelmingly agreed that gun control is an important issue and did not believe their opinions would change (Figure 2C). This highlights how passionate medical students are about gun ownership and violence issues consistent with broader polling that a majority of young adults believe gun legislation should be an immediate priority of congress. ${ }^{7}$ Over $80 \%$ of respondents in our study favored enhanced background checks, restricting high power rifles for military use, further restrictions on handgun ownership, and that illegal firearm sales should be punished more than illicit drug sales (Figure 2C). Overall, medical students in our survey favored increased restrictions on firearm ownership more so than young adults in a national poll. ${ }^{7}$ Our sample may be limited as the majority of students are from an urban city and tend to be more liberal-leaning, however, this may also highlight that those entering the medical field feel more strongly about gun safety issues. The overwhelming support of these issues and that over $90 \%$ of respondents identified gun control as an important issue suggests that medical students might support increasing curricular requirements on these and related topics

There was a major disconnect, however, when it came to the clinical application of gun control policy. Respondents were given nine different scenarios and asked to assess whether they would report a patient to federal authorities. Overall, students favored not reporting in most scenarios (mean $=30.5$; Figure $2 \mathrm{~B}$ ). Previous research suggests that both education and experience correlate with physicians' ability to perform more accurate risk assessments. ${ }^{4} \mathrm{~A}$ comprehensive review of medical education programs sought to identify those that provide firearm training and education for healthcare providers and were only able to identify four programs that met their criteria. ${ }^{3}$ Frank et al 2006 found that only $12 \%$ of surveyed medical students felt they had received "extensive" firearm safety training by the end of their medical school education. ${ }^{2}$ Therefore, it is unsurprising that medical students lack the education and experience to make risk assessments and decide when to report or not. A lack of training for firearm safety and injury prevention in undergraduate medical education puts future physicians at a disadvantage in performing adequately and confidently in these areas later; without repetition and reinforcement, future physicians report low rates of confidence on counseling on these issues. ${ }^{10}$

When assessing medical students' knowledge about current gun violence law, only $36 \%$ were able to answer at least seven out of ten questions correctly (mean: 5.9; SD 1.7; Figure 2A). Only slightly more than one-third of medical students sampled received a passing score, indicating a dearth of knowledge surrounding gun control issues. As stated earlier, health care training programs at all levels, including medical school, are lacking education on firearm 
violence. ${ }^{4,5}$ As firearm violence will remain a public health issue for years to come, it is paramount that medical education at all levels begins to address these gaps.

Both Washington University Medical School (St. Louis) and Harvard Medical School have begun the process of incorporating gun violence prevention programs into undergraduate medical education.

At Washington University, the course has been framed through a public health lens and includes teachings from community organizations to help reduce firearm injuries and violence. ${ }^{4}$ At Harvard Medical School, firearm, suicide, and homicide risk-assessment education are being worked into preclinical and clinical courses and clinical psychiatry clerkships. ${ }^{4}$ A multidisciplinary approach may be a good way to ensure that the gains made by training and interventions prove to have lasting effects. Other programs have used a short 20-minute lecture aimed at improving firearm counseling. They found that students who received the lecture had more confidence in counseling and improved their knowledge on risk factors for firearm violence immediately following the lecture, however, without any further teaching these improvements weakened at 6 months. ${ }^{5}$ They also found that physicians were more likely to provide firearm counseling to patients if prevention education is repeated at multiple levels (undergraduate, residency, continuing medical education) of training. ${ }^{5}$ Further research on curriculum and the proper way of incorporating these issues into current medical education is called for.

This paper is limited by sampling a small, homogeneous population that may not be generalizable. As discussed, the population was primarily white, living in an urban setting, which may explain why many respondents identified as liberal and/or Democrats. This paper does not account for potential differences in firearm violence within the curriculums of the medical schools surveyed, however, as respondents were primarily from a single institution it is likely any teaching in the current curriculum will be overrepresented. This study fails to address many of the nuances of firearm violence including suicide and mass shootings, but it highlights that medical education is currently lacking in an important public health issue that medical students are interested in learning more about.

\section{Conclusion}

Gun violence is an important public health issue that physicians will confront for the foreseeable future. Despite the relevance of this issue, medical education is generally lacking. In our survey of current medical students, many feel strongly about gun control issues and are unlikely to change their opinions; however, less than $40 \%$ successfully "passed" a short quiz about current firearm restrictions. This dearth of knowledge illustrates a gap in our current training of healthcare professionals that should be addressed through curriculum changes at all levels of training.

\section{References}

"Citizen's Guide to Federal Firearm Laws-Summary." NRAILA.org. National Rifle Association Institute for Legislative Action. 24, Mar. 2004. doi: https://doi.org/10.1016/j.ajem.2020.07.062

Frank, E., J.S. Carrera, J. Prystowsky, and A. Kellermann. 2006. Firearm-related Personal and Clinical Characteristics of US Medical Students. Southern Medical Journal. 99:216-225. doi: 10.1097/01.smj.0000202688.06133.f0.

Goldstein, A.O., A.J. Viera, J. Pierson, K.K. Barnhouse, J.A. Tulsky, and B.D. Richman. 2015. Physician Beliefs about Physical and Mental Competency of Patients Applying for Concealed Weapon Permits. Behavioral Sciences \& the Law. 33:238-245. doi: 10.1002/bsl.2169.

Gondi, S., A. Pomerantz, and C. Sacks. 2019. Extreme Risk Protection Orders: An Opportunity to Improve Gun Violence Prevention Training. Academic Medicine. 94:1649-1653. doi: 10.1097/ACM.0000000000002935.

Kwong, J.Z., J.M. Gray, L. Rein, Y. Liu, and M.D. Melzer-Lange. 2019. An educational intervention for medical students to improve self-efficacy in firearm injury preventioncounseling. Injury Epidemiology. 6:27-9. doi: 10.1186/s40621019-0201-3.

Neustadtl, A.L., Kaneb, A., Neustadtl, A.D., and Milzman, D. Emergency department providers knowledge, attitudes, and beliefs towards firearm ownership and reporting. The American Journal of Emergency Medicine. 27, July 2020

“NPR/PBS NewsHour/Marist Poll of 1,011 National Adults.” NPR/PBS NewsHour/Marist. April 2018.

Puttagunta, R., T. Coverdale, and J. Coverdale. 2016. What is Taught on Firearm Safety in Undergraduate, Graduate, and Continuing Medical Education? A Review of Educational Programs. Acad Psychiatry. 40:821-824. doi: 10.1007/s40596-016-0490-6.

Smith, Tom W., Davern, Michael, Freese, Jeremy, and Morgan, Stephen, General Social Surveys, 1972-2018 [machinereadable data file] /Principal Investigator, Smith, Tom W.; Co-Principal Investigators, Michael Davern, Jeremy Freese, and Stephen Morgan; Sponsored by National Science Foundation. Chicago: NORC, 2018: NORC at the University of Chicago [producer and distributor]. Data accessed from the GSS Data Explorer website at gssdataexplorer.norc.org. This GSS Data Explorer output was created by Karen Araiza on 2020-05-28.

Van Sparrentak, M., T. Chang, A.L. Miller, L.P. Nichols, and K.R. Sonneville. 2018. Youth Opinions About Guns and Gun Control in the United States. JAMA Pediatrics. 172:884-886. doi: 10.1001/jamapediatrics.2018.1746. 